\title{
Avaliação da precisão temática de composições de NDBI, NDVI, NDWI
}

\author{
Evaluation of thematic precision NDBI, NDVI, NDWI composition.
}

Júlio César Cotrim Moreira Filho ${ }^{1}$

João Rodrigues Tavares Junior ${ }^{2}$

${ }^{1}$ INCRA-Instituto Nacional de Colonização e Reforma Agrária. Rua E, s/n-Centro Político Administrativo, CEP 78.050-970, Cuiabá-MT-Brasil.

${ }^{2}$ Departamento de Engenharia Cartográfica, Universidade Federal de Pernambuco-UFPE. Av. Acadêmico Helio Ramos, s/n, Campus Joaquim Amazonas, 2 and., CEP 50.740-530. Recife-PE, Brasil.

jjcccmmff@gmail.com, joaoufpe@gmail.com

Recebido em 03/03/2015 - Aceito em 21/03/2016

Received on 03/03/2015 - Accepted on 03/21/2016

\begin{abstract}
RESUMO - Este artigo trata de experimentos usando composições de índices físicos NDVI (Normalized Difference Vegetation Index), NDWI (Normalized Difference Water Index), NDBI (Normalized Difference Built-Up Index), para avaliar a precisão temática do classificador Máxima Verossimilhança (MAXVER) usando exatidão global, índice kappa e teste Z. A área de estudo foi o entorno da Lagoa Olho D’Água localizada em Jaboatão dos Guararapes-PE. Foram usadas duas cenas TM LANDSAT-5, órbita-ponto 214-066, de 17/03/20111 e 29/09/2011, do DGI-INPE (Divisão de Geração de Imagens do Instituto Nacional de Pesquisas Espaciais), e todo o processamento realizado no SPRING 5.0.6. Os resultados indicam que apenas usando índices físicos substituindo composições RGB, a acurácia temática é muito degradada. A classificação MAXVER da composição NDBI-TM4-TM-3 e IHS obtiveram bons resultados em acurácia temática, demonstrando que o método de combinações de índices físicos e composições RGB podem ser usados para melhorar os resultados da classificação MAXVER.
\end{abstract}

Palavras-chave: avaliação de composições, índices físicos, precisão temática.

ABSTRACT - This article deals with experiments using physical indexes compositions NDVI (Normalized Difference Vegetation Index), NDWI (Normalized Difference Water Index), NDBI (Normalized Difference Built-Up Index) to evaluate the thematic accuracy of the classifier Maximum Likelihood (MAXVER) using accuracy global, kappa and test Z. The study area was around the Lagoa Olho D'Agua located in Jaboatão Guararapes-PE. We are used two scenes TM Landsat-5, orbit-point 214-066 of 17/03/20111 and 29/09/2011, the DGI-INPE (Imaging Division of the National Institute for Space Research), and all processing performed in SPRING 5.0.6. The results indicate that only using physical indices replacing RGB compositions, the thematic accuracy is very degraded. The MAXVER classification of NDBI-TM4-TM-3 and IHS composition obtained good results in thematic accuracy, demonstrating that this simple method of physical indexes combinations and RGB compositions can be used to improve the results of MAXVER rating.

Keywords: Review compositions, physical indices, thematic accuracy.

\section{INTRODUÇÃO}

Os índices físicos são normalmente usados separadamente e não formando combinações RGB (ZHA, et al., 2003; HE et al., 2010; JIANJU et al., 2005; CHEN et al., 2006; QIAN et al., 2007; LEE et al. 2011; LIU e ZHANG, 2011; JIANJU et al., 2005). Experimentos usando combinações RGB de índices físicos para avaliar o comportamento da precisão temática de classificadores supervisionados tem sido realizados e obtidos resultados promissores (SCOTT et al., 2014; FRANÇA et al., 2012). $\mathrm{O}$ índice kappa e exatidão global permitem aferir em imagens e mapas temáticos um índice de qualidade para a classificação de imagens supervisionadas (CONGALTON e MEAD, 1983). O classificador supervisionado de máxima verossimilhança (MAXVER) pode ser usado no SPRING onde a coleta das amostras possibilitam calcular a matriz de confusão e índice kappa (MARCELINO et al.,
2009). A Tabela 1 mostra alguns importantes trabalhos sobre a aplicação de classificadores e múltiplos índices físicos destacando os autores, sensor, bandas, método, valor de kappa obtido e a escala.

A área de estudo (Figuras 1 e 2), entorno da Lagoa Olho D'Água, foi escolhida por apresentar alvos contínuos de dimensões que permitem estudos espaciais e espectrais usando sensores de média resolução. Os alvos são água do mar, águas abrigadas da Lagoa Olho D’Água, vegetação de mangue e de restinga, coqueirais, área urbana, e setores de misturas destes alvos em áreas de transição. Os solos expostos são compostos por terraços arenosos das praias e do cordão litorâneo do Paiva, sedimentos lamosos, relevo predominantemente plano, e recifes de arenito próximos do litoral, enquanto a área urbana apresenta rede de estradas pavimentadas e vicinais, rodovias de várias faixas, área verticalizada e ocupações irregulares. A região também apresenta um 
sistema de pequenas lagoas e terrenos alagadiços, canais artificiais e naturais (MACEDO, 2010).

Tabela 1 - Trabalhos sobre aplicação de múltiplos índices físicos e classificadores.

\begin{tabular}{|c|c|c|c|c|c|}
\hline AUTOR & SENSOR & BANDAS & MÉTODO & КАРPA & ESCALA \\
\hline Zha et al. (2003) & TM & $3,4,5$ & NDVI, NDBI, MAXVER & $92,6 \%$ para o NDBI & $1: 150000$ \\
\hline $\begin{array}{l}\text { Jianjun et al. } \\
\text { (2005) }\end{array}$ & TM, ETM + & $3,4,5$ & NDBI, MAXVER & $98,96 \%$ e $78,75 \%$ & $1: 150000$ \\
\hline Chen et al. (2006) & TM, ETM + & 7 bandas & NDVI, NDWI, NDBI, NDBaI & $92,19 \%$ e $87,04 \%$ & $1: 150000$ \\
\hline Qian et al. (2007) & $\mathrm{ETM}+$ & 6 bandas & $\begin{array}{l}\text { NDBI, MAXVER, } \\
\text { Imagem Orientada a Objeto }\end{array}$ & $70,89 \% ; 89,33 \%$ & $1: 150000$ \\
\hline Zhang et al. (2009) & TM, ETM + & $3,4,5,6$ & LST, NDVI, NDBI & $\begin{array}{l}\text { Correlação de } 0,87 \text { e } 074 ; \\
0,29 \text { e } 0,07, \text { p/ LST-NDBI }\end{array}$ & $\begin{array}{l}1: 600000 \\
1: 150000\end{array}$ \\
\hline Liu e Zhang (2011) & $\begin{array}{l}\text { TM, } \\
\text { ASTER }\end{array}$ & $\begin{array}{l}\text { 7, TM, L1A } \\
\text { ASTER }\end{array}$ & LST, NDVI, NDBI, & $\begin{array}{l}\text { Correlação de }-0,41 \text { e } 0,71 \\
\text { para LSTNDVI e LST- } \\
\text { NDBI; UTFVI }\end{array}$ & $\begin{array}{l}1: 600000 \\
1: 150000 \\
1: 450000\end{array}$ \\
\hline Lee et al. (2011) & $\mathrm{TM}, \mathrm{ETM}+$ & $3,4,5,6$ & $\begin{array}{l}\text { BT e Árvore de decisão, } \\
\text { NDVI, NDWI, NDBI, NDBaI }\end{array}$ & $\begin{array}{l}90 \% \text { para água e área urbana } \\
\text { e relação direta entre BT e } \\
\text { área urbana }\end{array}$ & $1: 150000$ \\
\hline
\end{tabular}

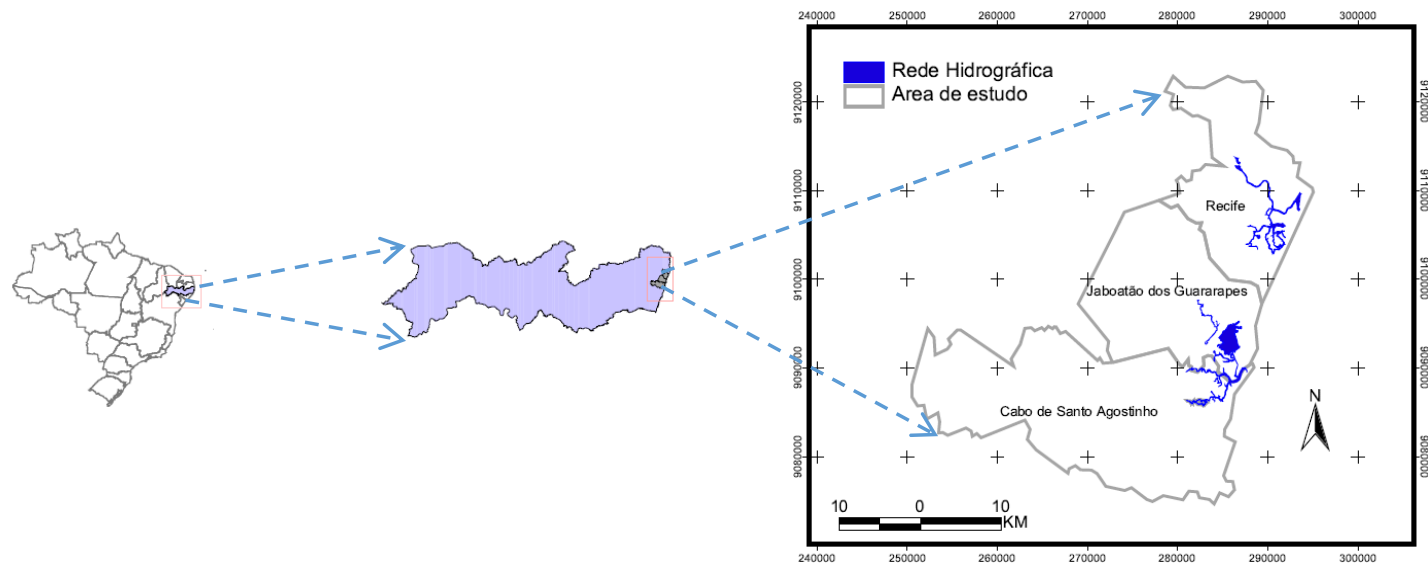

Figura 1 - Localização da área de estudo.
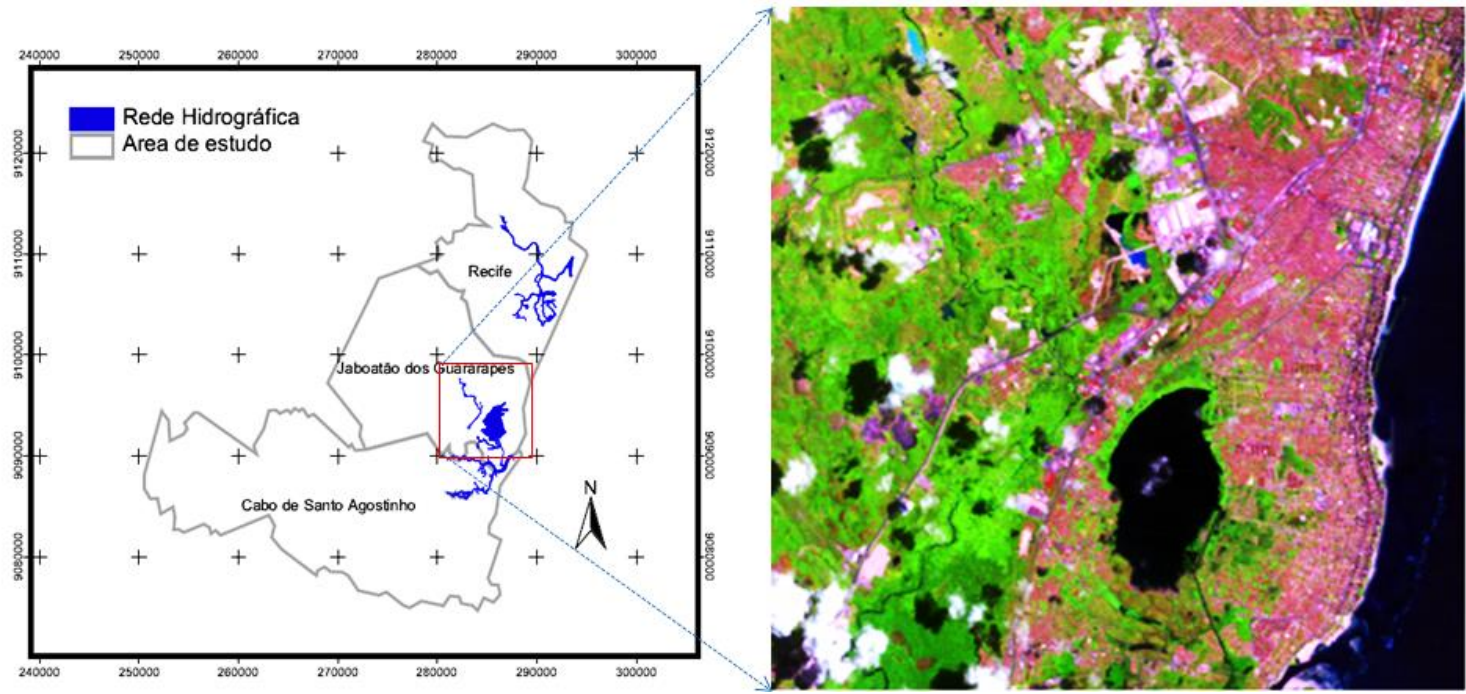

Figura 2 - Localização e imagem da área de estudo. 
O objetivo do trabalho é avaliar a precisão temática do classificador Máxima Verossimilhança (MAXVER) usando exatidão global, índice kappa e teste $\mathrm{Z}$ em composições de índices físicos NDVI (Normalized Difference Vegetation Index), NDWI (Normalized Difference Water Index), NDBI (Normalized Difference Built-Up Index).

A Equação 1 mostra o algoritmo da máxima verossimilhança (MAXVER) em que $P c$ é a probabilidade de o vetor $X$ ser assinalado; $V c$ a matriz de covariância da classe c contemplando todas as bandas; $\operatorname{Det}\left(V_{c}\right)$ é o determinante da matriz de covariância $V c ; X$ o vetor de medidas dos pixels desconhecidos; $M c$ vetor das médias para cada classe $c$; e $T$ é a matriz transposta (ESTEHMAN e CZAPLEWSKI, 1998).

$$
P c=\left[-0,5 \log _{e}\left(\operatorname{Det}\left(V_{c}\right)\right)\right]-\left[0,5\left(X-M_{c}\right)^{T}\left(V_{c}\right)^{-1}\left(X-M_{c}\right)\right]
$$

Para o cálculo de Kappa é necessário construir a matriz de confusão que exibe a quantificação de acertos e erros quanto à classificação realizada para cada alvo, sendo expostos os valores de verdade do terreno como colunas e a classificação nas linhas (JUNIOR e BACANI, 2011). Após a construção da matriz de confusão, torna-se possível o cálculo de Kappa (Equação 2), demonstrada em Junior e Bacani (2011), inicialmente apresentada em Cohen (1960), onde " $n$ " é o tamanho da amostra, "r" o número de alvos, " $\mathrm{x}_{\mathrm{ii}}$ " valores da diagonal principal, " $\mathrm{x}_{\mathrm{i}+}$ " valores totais das linhas, " $\mathrm{x}_{\mathrm{i}+\mathrm{i}}$ " valores nas colunas.

$$
\hat{k}=\frac{n \sum_{i=1}^{r} x_{i i}-\sum_{i=1}^{r} x_{i+} x_{+i}}{n^{2}-\sum_{i=1}^{r} x_{i+} x_{+i}}
$$

O maior valor para o coeficiente kappa é referente a uma classificação perfeita, e onde, a matriz de confusão será representada apenas por elementos da diagonal principal. Neste caso (SOUTO, 2000) o cálculo de kappa será dado pela Equação 3.

$$
\hat{k}=\frac{n^{2}-\sum_{i=1}^{r} x_{i+} x_{+i}}{n^{2}-\sum_{i=1}^{r} x_{i+} x_{+i}}=1
$$

Com isso o maior valor a ser descrito por kappa é $\mathrm{k}=1$ porém quando todos os pontos forem incorretamente classificados, têm-se o caso oposto, de menor valor para kappa (Equação 4).

$$
\hat{k}=\frac{-\sum_{i=1}^{r} x_{i+} x_{+i}}{n^{2}-\sum_{i=1}^{r} x_{i+} x_{+i}}
$$

CONGALTON e GREEN (1998) apresentam a equação do teste $\mathrm{Z}$ que trata da significância estatística da diferença entre dois índices Kappa.

As equações (5), (6) e (7) apresentam índices estudados NDVI, e NDBI. Maiores detalhes sobre estes índices podem ser vistos em FRANÇA, TAVARES JUNIOR e MOREIRA FILHO (2012) e nos autores da Tabela 1.

NDVI $=(\rho \operatorname{ivp}-\rho v m) /(\rho \operatorname{ivp}+\rho v m)$.

NDWI $=(\rho v d-\rho i v p) /(\rho v d+\rho i v p)$.

$\mathrm{NDBI}=(\operatorname{\rho ivm}-\rho \operatorname{ivp}) /(\operatorname{\rho ivm}+\rho \operatorname{\rho ivp})$.

Onde pivp é a refletância no infravermelho próximo, $\rho v m$ a refletância no vermelho, $\rho v d$ a reflectância na faixa do verde e pivm a reflectância no infravermelho médio.

\section{MATERIAIS E MÉTODOS}

O fluxograma da Figura 3 mostra o método usado com os dados de entrada, pré-processamento (delimitação da área de estudo, correção geométrica nos mosaicos GEOCOVER 2000, em UTM/WGS84; modificação da resolução espacial de $30 \mathrm{~m}$ para $15 \mathrm{~m}$; cálculo dos índices; transformação RGB-IHS); processamento (6 composições para cada cena, total de 12 composições; classificações MAXVER de todas as composições; interpretação visual das classificações; cálculo da exatidão global, kappa e teste Z) e dados de saída (análise da estatística global, kappa, teste $\mathrm{Z}$ das classificações; conclusões e discussão). As classes foram água, vegetação, área urbana, solo exposto e vegetação, e a MAXVER da imagem TM5-TM4-TM3, foi usada como referência visual na interpretação dos alvos.

Os resultados de NDVI, NDWI e NDBI foram obtidos a partir de dados de reflectância com correção atmosférica (DOS - Dark Object Subtraction) baseados em GURTLER et al. (2005). 


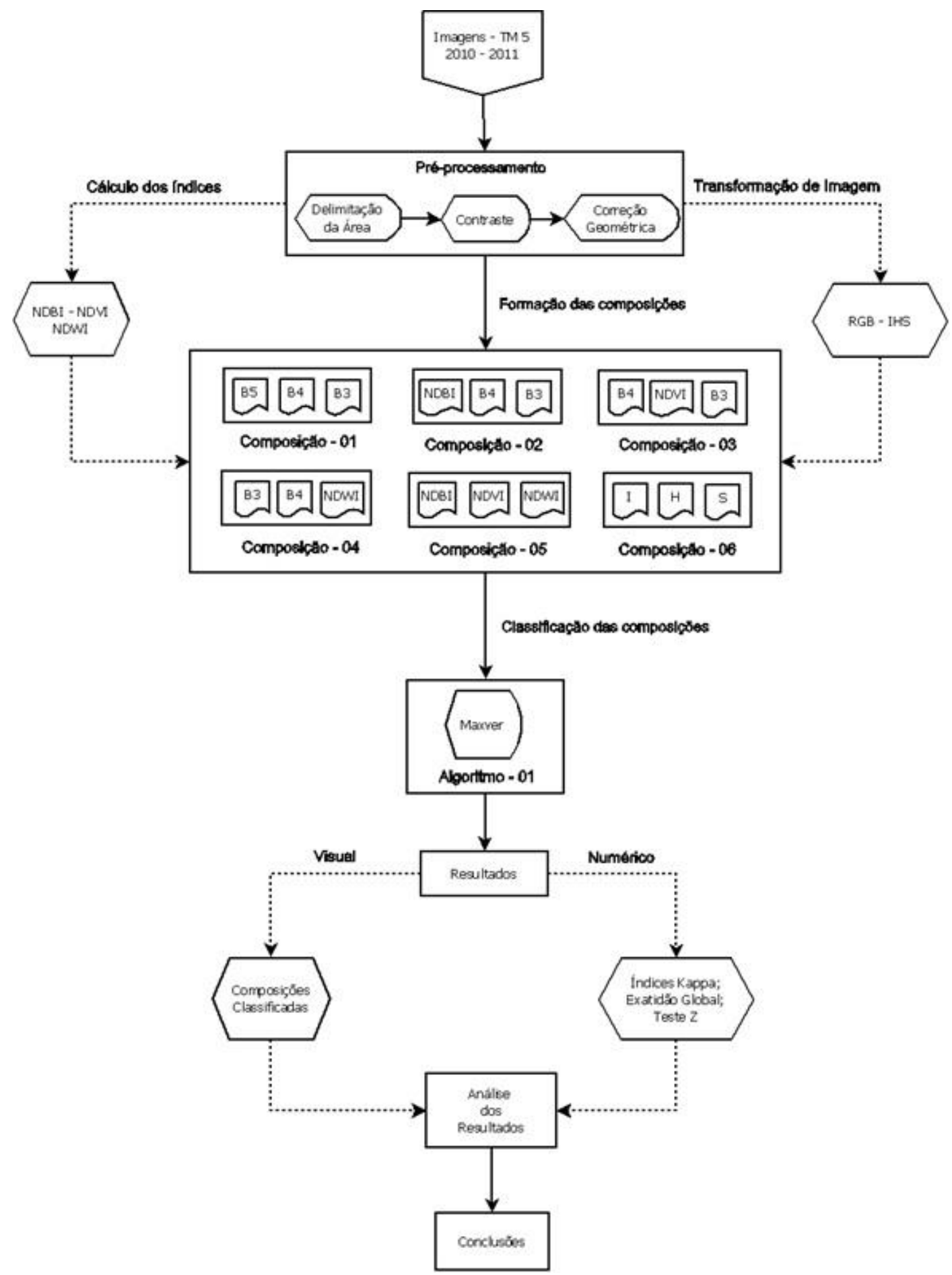

Figura 3 - Fluxograma do método.

\section{RESULTADOS}

A Figura 4 (17/03/2011) mostra a imagem NDBITM4-TM3, tendo a área urbana a separação visual de alvos semelhante a imagem TM5-TM4-TM3. A imagem TM5-NDVI-TM3 destacou melhor a vegetação, linha de costa, principais vias, porém, as cores da área urbana e nuvens-sombras não foram bem separadas. Nas outras composições os resultados não foram bons, ocorrendo confusão entre alvos. Outro comportamento das composições foi o destaque dos ruídos da varredura linear pushbroom do TM, com faixas transversais a direção da órbita do sistema satélite LANDSAT-5. Em todas as imagens o norte está na direção da parte de cima.

A Figura 5 (29/09/2011) mostra a imagem NDBITM4-TM3 separando a linha de costa, nuvens-sombras, borda da Lagoa Olho D’Água, recifes de arenito. Na Figura 5 a imagem TM5-TM4-NDWI, delimitou melhor as rodovias principais, linha de costa, borda da lagoa Olho D’Água, e estirâncio (faixa de areia da praia exposta pela 
baixa mar); enquanto a imagem TM5-NDVI-TM3 define bem a rede principal e secundária de rodovias e linha de costa.

Na Figura $6(17 / 03 / 2011)$ a linha de costa e margens da Lagoa Olho D'Água estão bem definidas em todas as imagens, exceto as margens da lagoa na classificação MAXVER do NDBI-NDVI-NDWI que se misturou a vegetação. A imagem MAXVER do IHS na Figura 6 separou o estirâncio, apesar de misturar diferentes subclasses de área urbana. Na Figura 6 o Rio Jaboatão (canto inferior esquerdo, sudoeste: "r.J."), e pouco visível na imagem MAXVER da composição TM5-TM4-TM3, e na imagem TM5-NDVI-TM3.

Na Figura 7 (29/09/2011) a linha de costa está bem delineada, porém os recifes de arenito não estão bem separados, talvez em função da menor transparência da água e maior incidência de ondas, estando a maré baixa.

$\mathrm{Na}$ Figura 7 da classificação MAXVER da combinação NDBI-NDVI-NDWI o Rio Jaboatão está bem separado da vegetação vizinha nas margens, enquanto nas outras as imagens o mesmo rio não está visível. Também na Figura 7 a imagem MAXVER do IHS o estirâncio está bem definido, embora haja intensa confusão entre área urbana e nuvens-sombras. A MAXVER da combinação NDBI-NDVI-NDWI mostra a BR-101 que cruza a imagem a partir do canto superior direito até o canto inferior esquerdo (NE-SW), mas há mistura de água da Lagoa Olho D’Água e área urbana (Figura 7).

Comparando visualmente as Figuras 6 (17/03/2011, período com tendência a maior chuva) e a $7(29 / 09 / 2011$, período com tendência a menos chuva), observa-se significativas diferenças na classificação MAXVER, principalmente entre as classificações MAXVER do IHS, onde a rede urbana se confunde com nuvens-sombras.

Na Figura 8 as imagens NDBI, NDVI, NDWI de 17/03 e 29/09 de 2011 mostram intensas as marcas do ruído da varredura TM na superfície marinha. Na imagem de 17/03, Figura 8, a Lagoa Olho D’Água mostra intenso NDVI, época de pluviometria acumulada de $2522,1 \mathrm{~mm}$, enquanto em 29/09/2011 choveu $43 \mathrm{~mm}$ (APAC, 2015).

O NDVI da Figura 8 (17/03 e 29/09/2011), separou bem a linha de costa (maré em torno de $0,1 \mathrm{~m}$; DHN, 2015) e bordas da lagoa Olho D’Água. A área urbana e vegetação foram bem separadas apenas no setor leste da imagem correspondente ao litoral, considerando que as nuvens-sombras, muito frequentes nesta região, e dificultam a visualização da área urbana (Figura 8).

O NDVI de 17/03/2011 na Lagoa Olho D'Água na Figura 8 indica provável detecção de microalgas e vegetação flutuante, com maior proliferação em águas com lançamento de esgoto sem tratamento.

$\mathrm{Na}$ cena de 17/03 ocorreu a maré baixa de $0,4 \mathrm{~m}$, calculada para as coordenadas $\varphi=8^{\circ} 03,4^{\prime} ; \lambda=-34^{\circ} 52,1^{\prime}$, Porto do Recife-PE (DHN, 2015). Considerando a passagem do TM aproximadamente as $9 \mathrm{~h} 30$ (Tempo Legal, 12 h TMG), a maré estava ainda subindo e revelando mais faixa de areia das praias de Candeias, Piedade e Barra de Jangada, faixa que pode chegar até cerca de $60 \mathrm{~m}$ de largura em alguns trechos.
A Figura 9 apresenta os valores calculados para kappa e exatidão global para as seis composições de $17 / 03 / 2011$ e seis de 25/09/2011. Os melhores kappa foram obtidos para o IHS e NDBI-TM4-TM3. Na Figura 9 a imagem NDBI-NDVI-NDWI, para as duas datas, mostram valores menores que as outras composições, embora estejam acima de 0,8. A composição NDBINDVI-NDWI não apresenta contribuição na melhora da classificação MAXVER em relação a imagem MAXVER da TM5-TM4-TM3.

Na Figura 9 a maior precisão ocorre na MAXVER da imagem de referência TM5-TM4-TM3, com valores acima de 0,9, para 17/03 e 29/09 de 2011, e as variações relativas da exatidão, para as duas datas, ocorreram principalmente para kappa, enquanto a variação relativa da exatidão global mostra menor variação, exceto na NDBI-NDVI-NDWI.

Na Figura 9 os valores de kappa mostram variações menores, nas duas datas, para TM5-TM4-NDWI e IHS, e maior variação para NDBI-NDVI-NDWI. A exatidão global, de 17/03 e 29/09, pouco mudou para NDBI-TM4TM3, mas o kappa foi menor em setembro. Para 29/09 a diferença entre kappa e exatidão global foi maior que em março.

O teste " $Z$ " foi aplicado nas seis imagens de cada data (12 ao total), na Figura 10, que é dividida para o mês de março (17/03/2011) e setembro (29/09/2011) mostra valores $Z$ calculados de dois modos: um modo é a " 1 a composição" que usa o kappa da classificação MAXVER da TM5-TM4-TM3 e o compara ao kappa de cada uma das 12 composições. A segunda maneira é comparar o valor de " $Z$ " "entre as composições" que avaliou a concordância entre o kappa da MAXVER de cada uma das 12 composições e o kappa da MAXVER da imagem de referência TM5-TM4-TM3. Quanto mais afastado de zero (posição da imagem referência na Figura 10) for o valor de "Z", pior a concordância entre a classificação MAXVER de cada uma das 12 composições e a MAXVER da TM5-TM4-TM3.

Seguindo a linha do gráfico da Figura 10 denominada " 1 a composição" de 17/03 os melhores resultados de concordância no teste " $Z$ " foram obtidos para NDBI-TM4-TM3 e IHS, e o pior foi para NDBINDVI-NDWI. Em setembro o melhor valor de " $Z$ " foi para a imagem TM5-NDVI-TM3, e o pior TM5-TM4NDWI.

Na Figura 10 a linha "entre as composições" foi usada para avaliar a possibilidade de quedas de tendência na concordância; assim sendo, os piores resultados são da TM5-TM4-NDWI e NDBI-NDVI-NDWI para 17/03 e 29/09. A composição IHS mostrou bom resultado em concordância para ambos os meses.

A Tabela 2 agrupa todos os resultados kappa, exatidão global e teste $\mathrm{Z}$ com o objetivo de avaliar em conjunto qual a composição obteve melhor e pior resultado da classificação MAXVER das composições. Na Tabela 2 "M" indica o melhor resultado e "P" o pior resultado: a composição NDBI-TM4-TM3 e IHS apresentaram os melhores resultados de classificação, e a imagem NDBI-NDVI-NDWI os piores resultados. 
Tabela 2 - avaliação geral de kappa, exatidão global e teste $\mathrm{Z}$ de todas as classificações das composições.

\begin{tabular}{|c|c|c|c|c|c|}
\hline AVALIAÇÃO & $\begin{array}{c}\text { NDBI-TM4- } \\
\text { TM3 }\end{array}$ & TM5-NDVI-TM3 & TM5-TM4-NDWI & $\begin{array}{c}\text { NDBI-NDVI- } \\
\text { NDWI }\end{array}$ & I-H-S \\
\hline $\begin{array}{c}\text { Kappa e exatidão } \\
\text { global-Gráfico barra- } \\
\text { Março }\end{array}$ & $M$ & & & $P$ & $M$ \\
\hline $\begin{array}{c}\text { Kappa e exatidão } \\
\text { global-Gráfico barra } \\
\text { Setembro }\end{array}$ & $M$ & & $M$ & $P$ & $M$ \\
\hline $\begin{array}{l}\text { Variação de kappa } \\
\text { entre datas } \\
\text { Gráfico dispersão }\end{array}$ & & & $M$ & $P$ & $M$ \\
\hline $\begin{array}{l}\text { Variação de Exatidão } \\
\text { global entre datas. } \\
\text { Gráfico dispersão }\end{array}$ & $M$ & $M$ & & $P$ & \\
\hline $\begin{array}{c}\text { Teste Z: “1ª } \\
\text { composição" Março }\end{array}$ & $M$ & & & $P$ & $M$ \\
\hline $\begin{array}{c}\text { Teste } \mathrm{Z:} \text { : “1”a } \\
\text { composição” } \\
\text { Setembro }\end{array}$ & & $M$ & $P$ & & \\
\hline $\begin{array}{c}\text { Melhores resultados } \\
\text { de MAXVER das } \\
\text { composições }\end{array}$ & $M$ & & & & $M$ \\
\hline $\begin{array}{c}\text { Pior resultado de } \\
\text { MAXVER de } \\
\text { composição }\end{array}$ & & & & $P$ & \\
\hline
\end{tabular}




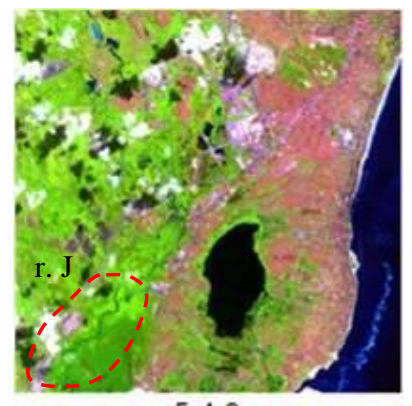

5-4-3

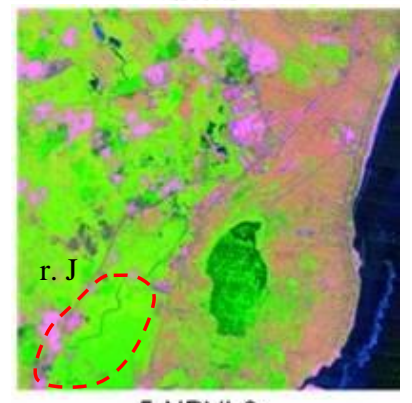

5-NDVI-3

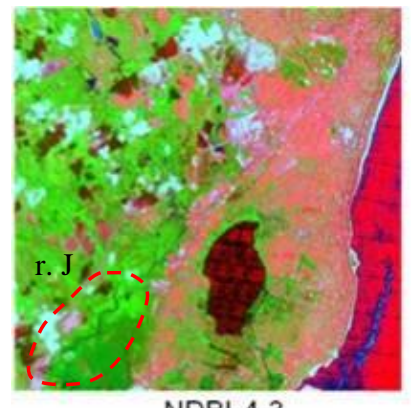

NDBI-4-3

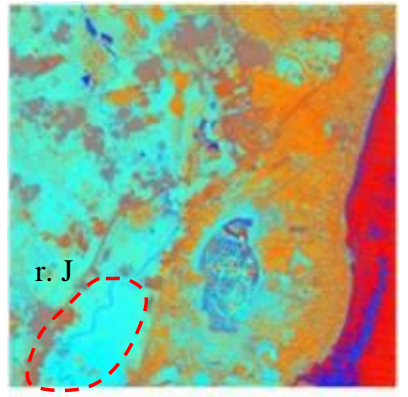

NDBI-NDVI-NDWI

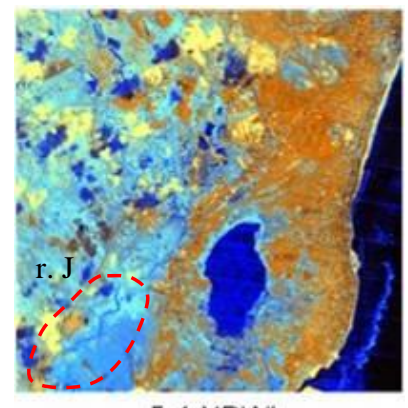

5-4-NDWI

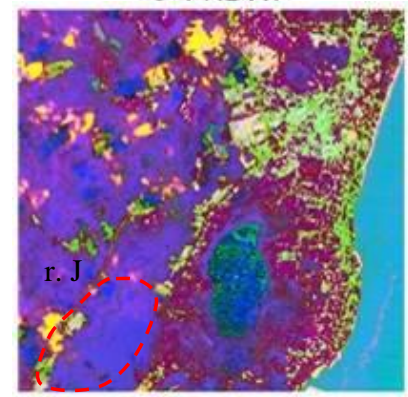

I-H-S

Figura 4 - Composições de 17/03/2011.

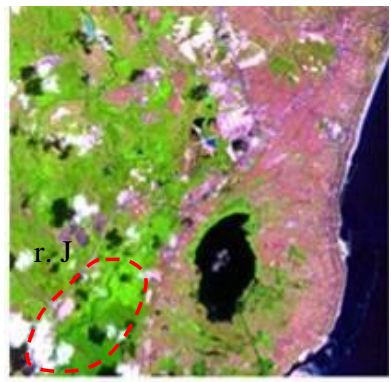

$5-4-3$

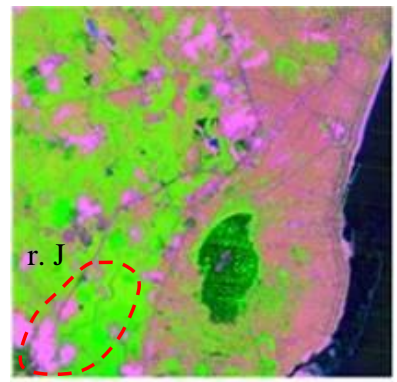

5-NDVI-3

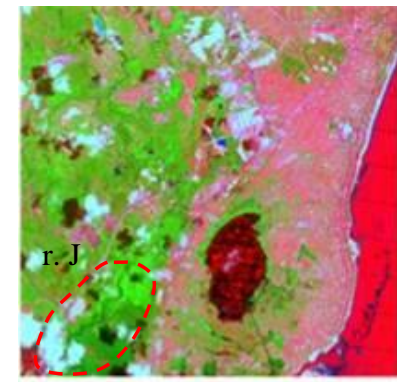

NDBI-4-3

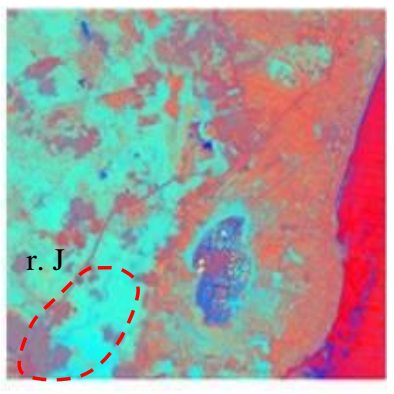

NDBI-NDVI-NDWI

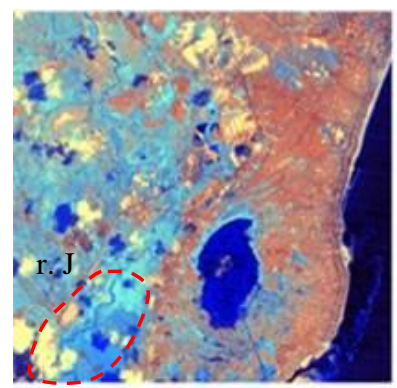

5-4-NDWI

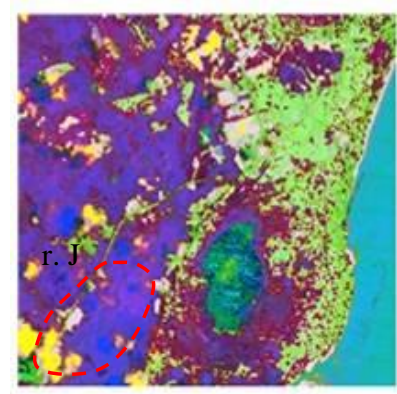

$\mathrm{I}-\mathrm{H}-\mathrm{S}$

Figura 5 - Composições de 29/09/2011. 


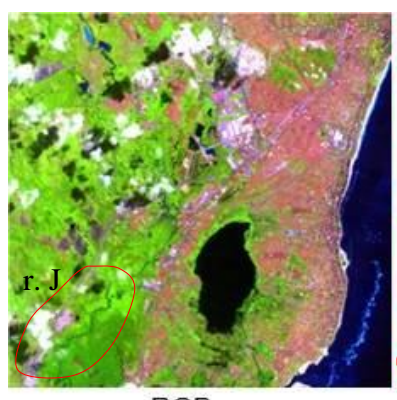

RGB
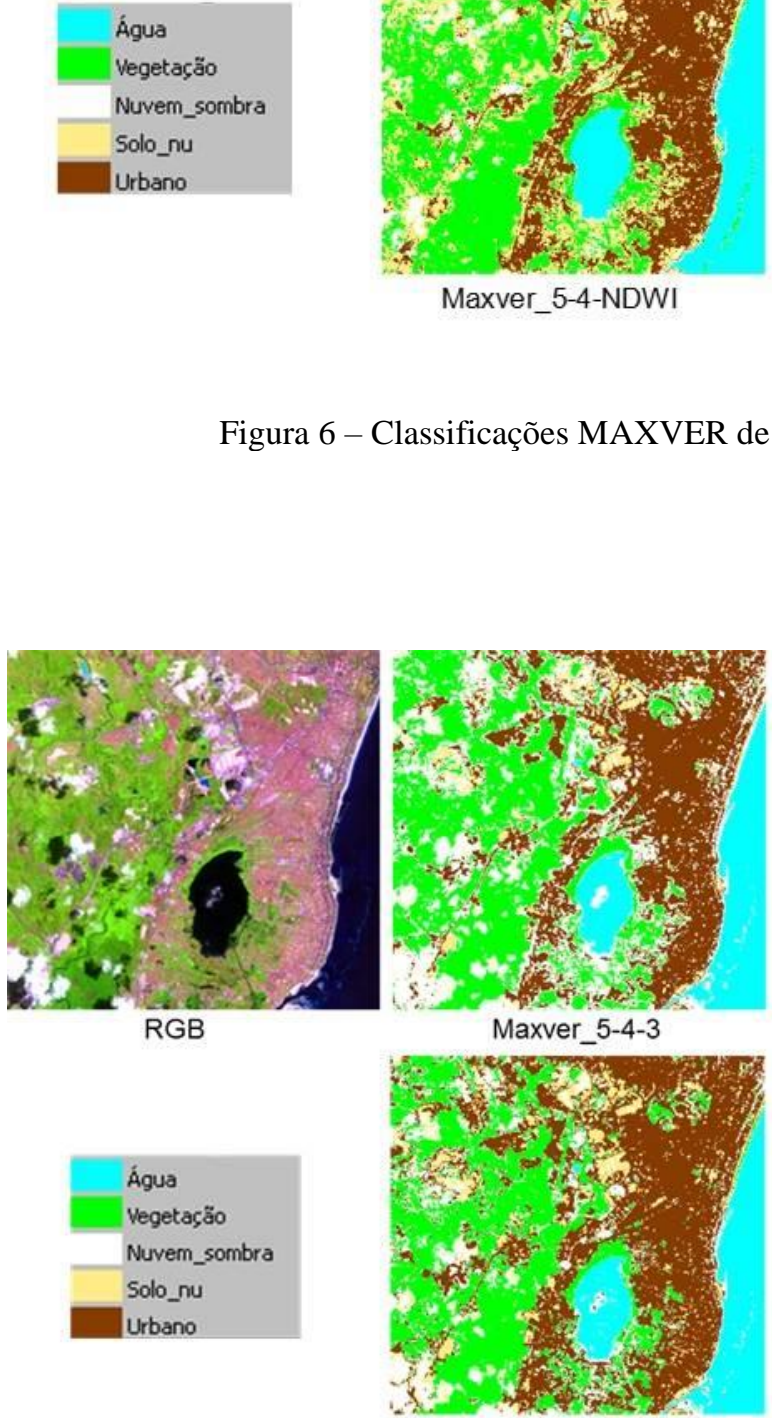

Maxver_5-4-NDWI

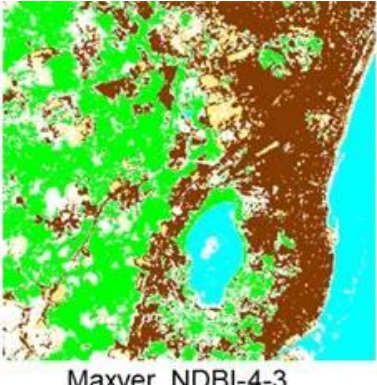

Maxver_NDBI-4-3

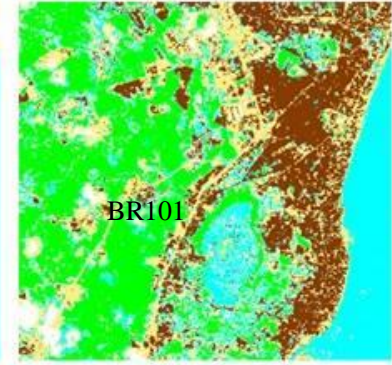

Maxver_NDBI-NDVI-NDWI
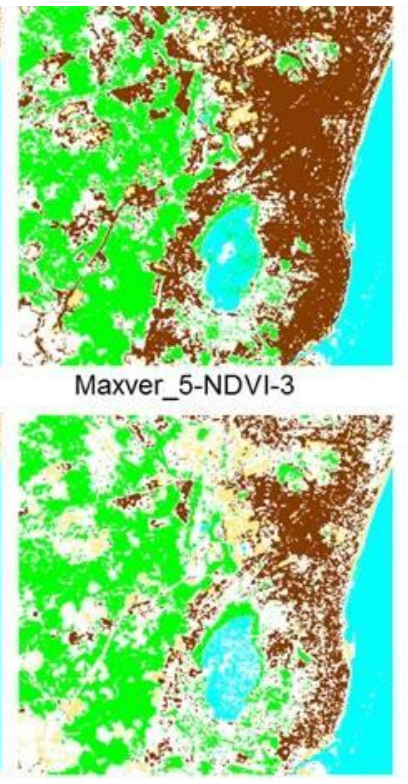

Maxver_I-H-S

Figura 7 - Classificações MAXVER de 29/09/2011 das composições da Figura 5. 


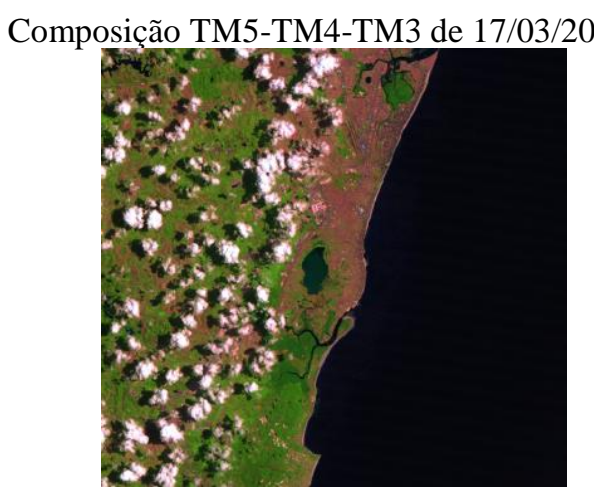

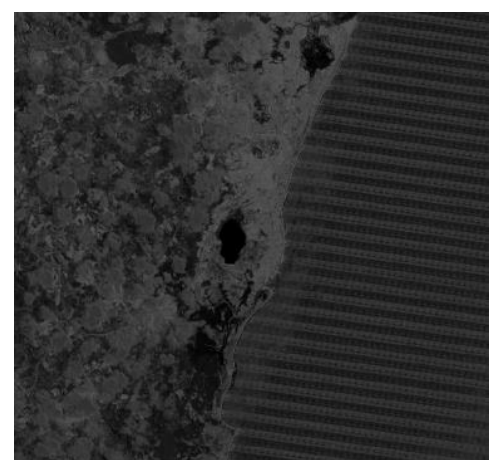

NDBI - 17/03/2011

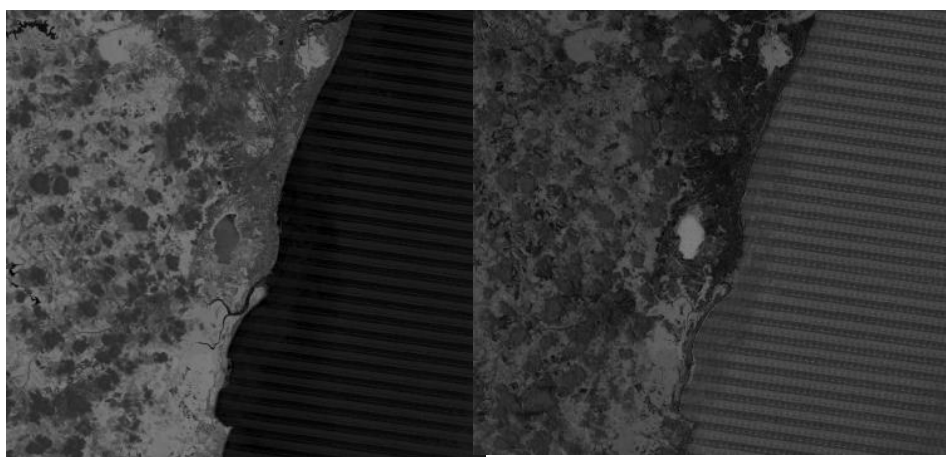

NDVI- 17/03/2011
NDWI- 17/03/2011

Composição TM5-TM4-TM3 de 25/09/2011
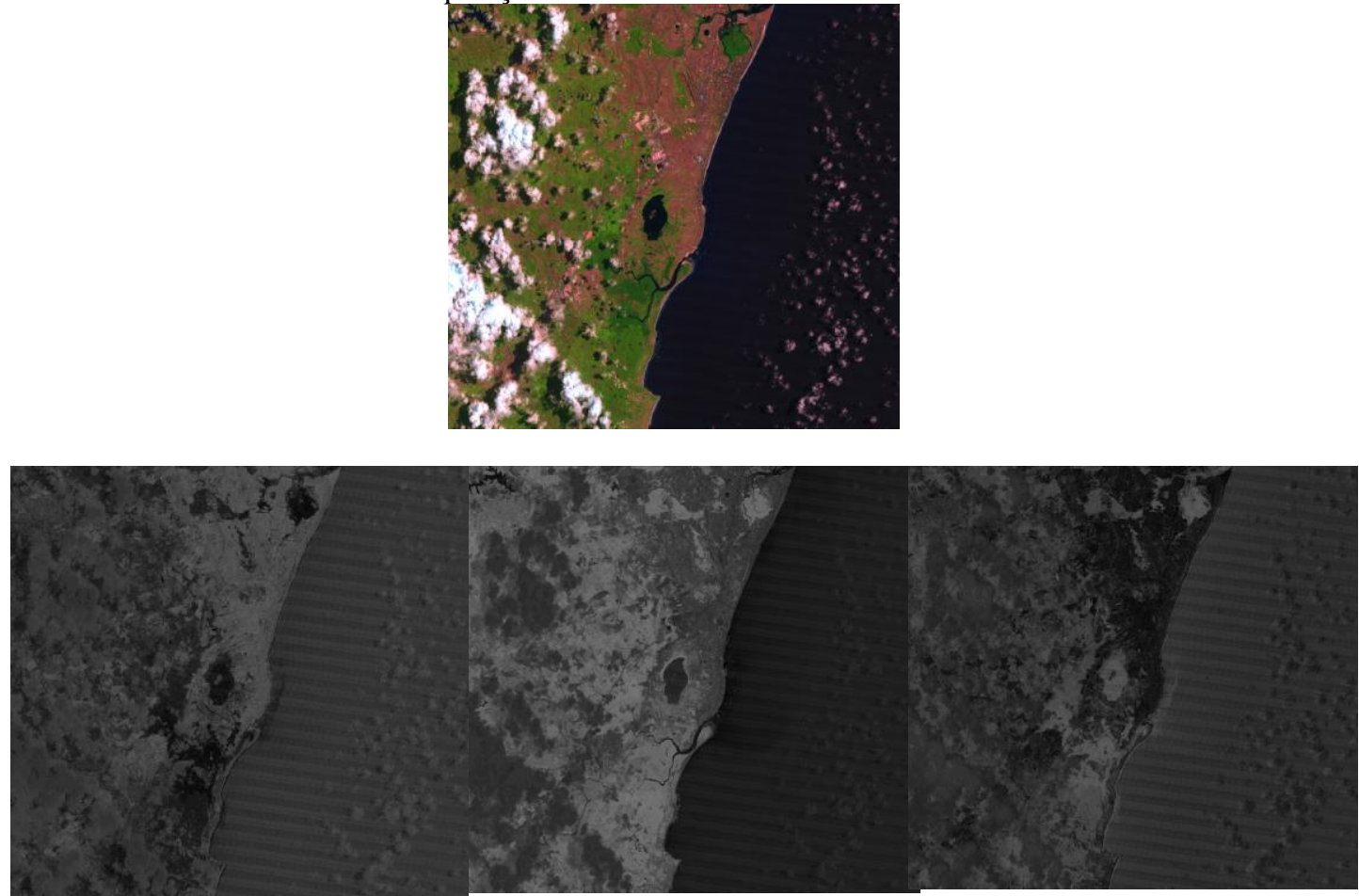

NDBI - 25/09/2011

NDVI- 25/09/2011

NDWI- 25/09/2011

Figura 8 - Índices físicos de março e setembro de 2011 

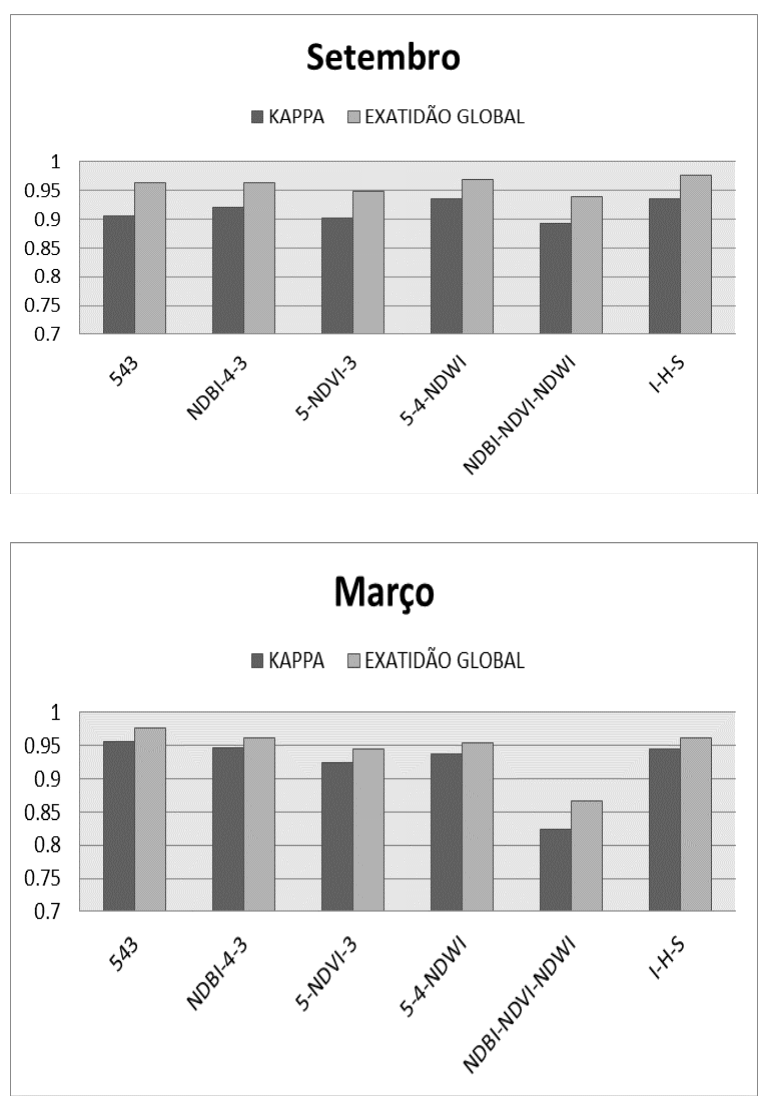

Figura 9 - Kappa e exatidão das 12 composições.

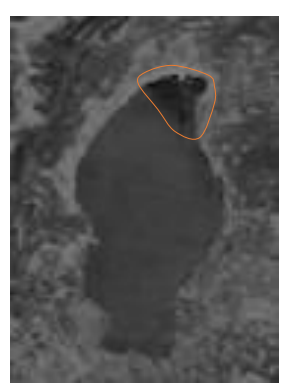

(a) NDVI

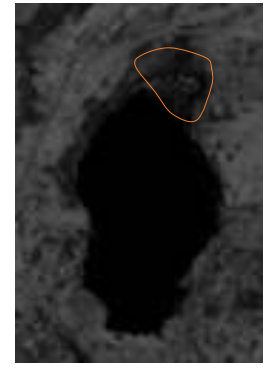

(b) NDBI

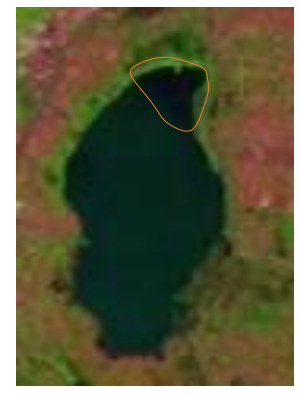

(c) TM543

Figura 11 - Índices e composição da extremidade norte da Lagoa Olho D’Água (17/03/2011).
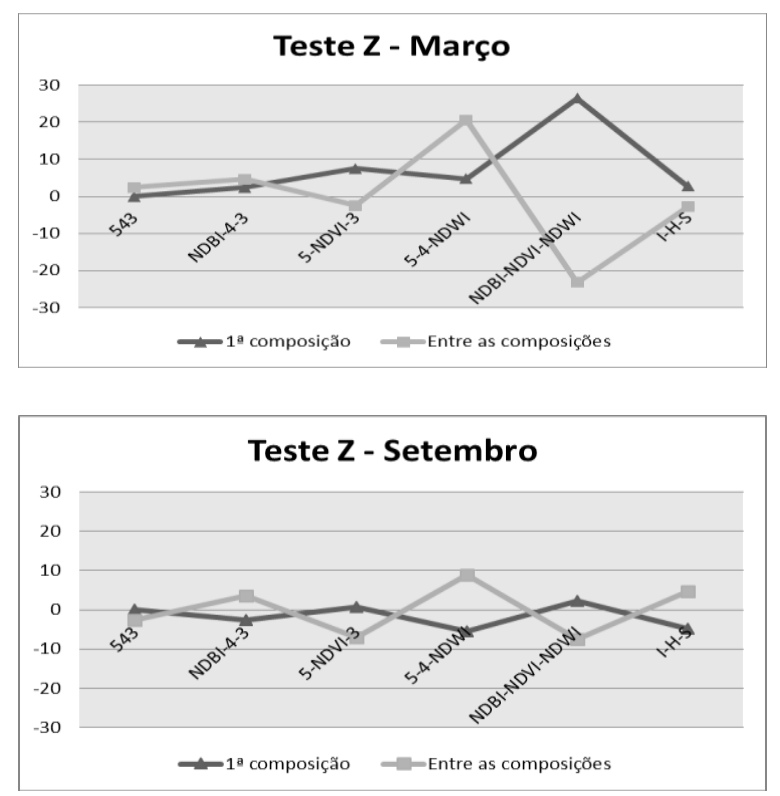

Figura 10 - Teste $\mathrm{Z}$ das 12 composições

\section{CONCLUSÃO E DISCUSSÃO}

A classificação MAXVER das composições NDBITM4-TM3 e IHS apresentaram as melhores precisões temáticas, demonstrando relativa utilidade para melhorar o índice kappa do classificador MAXVER em imagens multiespectrais de média resolução espacial. Feições lineares podem ser separadas usando as composições classificadas em MAXVER, a exemplo do Rio Jaboatão e BR-101 (TM5-NDVI-TM3), melhor valor de teste " $Z$ " para setembro; contorno da Lagoa Olho D’Água, recifes de arenito acima da linha d'água (TM5-TM4-NDWI, apesar desta composição ter o pior resultado no teste " $Z$ " para setembro), o estirâncio (NDBI-TM4-TM3, melhor " $Z$ " para o mês de março). Tanto o teste " $Z$ " da composição NDBI-NDVI-NDWI como o índice kappa e exatidão global (principalmente para setembro) apresentaram os piores resultados, não sendo recomendadas estas combinações para a melhora da precisão temática usando o classificador MAXVER. Por outro lado, comparando o teste " $Z$ ”, índice kappa e exatidão global do NDBI-NDVI-NDWI entre março e setembro, este último obteve melhor resultado, talvez devido a ser este o período mais seco que março, e separar melhor solo exposto (estirâncio) e principais rodovias. A classificação MAXVER do IHS, mesmo tendo índice kappa alto não evitou a visualização da mistura de subclasses de área urbana e também com nuvens-sombras. Apesar da classificação da composição NDBI-NDVI- 
NDWI obter um kappa relativamente menor que outras classificações de composições, esta composição de índices físicos foi adequada para separar o Rio Jaboatão. A interpretação visual, utilizada com os devidos cuidados, ainda é uma boa técnica de auxílio e supervisão para verificar falhas localizadas em imagens classificadas com MAXVER a partir de composições de índices físicos e RGB com índices kappa altos.

\section{REFERÊNCIAS}

APAC. Agência Pernambucana de Águas e Clima. Disponível em http://www.apac.pe.gov/ Consulta em fevereiro de 2015.

CHEN, X.; ZHAO, H.; LI, P.; YI, Z. Remote sensing image-based analysis of the relationship between urban heat island and land use/cover changes. Remote Sensing of Environment, Elsevier, v. 104, n. 2, p. 133-146, 2006.

COHEN, J. A coefficient of agreement for nominal scales. Educational and psychological measurement, v.20, n.1, p.37-46, 1960.

CONGALTON, R. G.; GREEN, K. Assessing the accuracy of remotely sensed data: principles and practices. New York: Lewis Publishers, 1998. 137p.

CONGALTON, R. G.; MEAD, R. A. A quantitative method to test for consistency and correctness in photointerpretation. Photogrammetric Engineering and Remote Sensing, v.49, n.1, p.69-74, 1983.

DHN. Diretoria de Hidrografia e Navegação. Centro de Hidrografia da Marinha. Banco Nacional de dados Oceanográficos. Disponível em: <http:www.mar.mil.br/dhn/chm/box-previsãomare/tabuas/>. Consulta em fevereiro de 2015.

ESTEHMAN, S. V.; CZAPLEWSKI, L. Design and Analysis for Thematic Map Accuracy Assessment: Fundamental Principles. Remote Sensing of Environment, v. 64, p. 331-344. 1998.

FRANÇA, A. F., TAVARES JUNIOR, J. R., MOREIRA FILHO, J. C. C. Índices NDVI, NDWI e NDBI como ferramentas do mapeamento temático do entorno da lagoa Olho D'Água em Jaboatão dos Guararapes. In: Anais... IV Simpósio Brasileiro de Ciências Geodésicas e Tecnologias da Geoinformação. p1-9. Recife. 2012.

Gürtler, S.; Epiphanio, J.C.N.; Luiz, A.J.B.; Formaggio, A.R. Planilha eletrônica para o cálculo da reflectância em imagens TM e ETM+ Landsat. Revista Brasileira de Cartografia, v. 57, p. 162-167, 2005.

LEE, L.; CHEN, L.; WANG, X.; ZHAO, J. Use of landsat tm/etm + data to analyze urban heat island and its relationship with land use/cover change. International
Conference on Remote Sensing, Environment and Transportation Engineering - RSETE, p. 922 - 927, 2011. Nanjing.

JUNIOR, C. A. S.; BACANI, V. M. Aplicação de diferentes métodos de classificação supervisionada de imagem landsat-5/tm na identificação de cana-de-açúcar. Curitiba: Anais XV Simpósio Brasileiro de Sensoriamento Remoto - SBSR, XV, p. 85-92, 2011.

JIANJUN, J.; JIE, Z.; HONG'AN, W.; LI, A.; HAILONG, Z.; LI, Z.; JUN, X. Land cover changes in the rural-urban interaction of xian region using landsat tm/etm data. Journal of Geographical Sciences, Science China Press, co-published with Springer, v. 15, p. 423430, 2005.

LIU, L.; ZHANG, Y. Urban heat island analysis using the Landsat tm data and aster data: A case study in Hong Kong. Remote Sensing, v. 3, n. 7, p. 1535-1552, 2011. ISSN 2072-4292.

MACEDO, O. G. de. Avaliação de classificadores em imagem de alta resolução. Área urbana de Jaboatão dos Guararapes- PE. Dissertação (Mestrado em ciências Geodésicas e Tecnologias da Geoinformação). Universidade Federal de Pernambuco. 100p. 2010.

MARCELINO, E. V., FORMAGGIO, A. R., MAEDA, E. E. Landslide inventory using image fusion techniques in Brazil., International Journal of Applied Earth Observation and Geoinformation. 11, p.181-191. 2009.

QIAN, J.; ZHOU, Q.; HOU, Q. Comparison of pixelbased and object-oriented classification methods for extracting built-up areas in aridzone. SPRS Workshop on Updating Geo-Spatial Databases with Imagery \& The 5th ISPRS Workshop on DMGISs, p. 163-171, 2007.

HE, C.; SHI, P.; XIE, D.; ZHAO, Y. Improving the normalized difference built-up index to map urban builtup areas using a semiautomatic segmentation approach. Remote Sensing Letters, v. 1, n. 4, p. 213-221, 2010. Disponível em: <http://www.tandfonline.com/doi/abs/10.1080/01431161. $2010.481681>$.

SCOTT, D., PETROPOUlO, G. P., MOXLEY, J., MALCOLM, H., Quantifying the physical composition of the urban morphology throughout wales based on the time series (1989-2011) analysis of Landsat TM/ETM+ images and supporting GIS data. Remote Sensing, 6, p.1173111752. 2014.

SOUTO, R. P. Segmentação de imagem multiespectral utilizando-se o atributo matiz. Dissertação (Mestrado em Sensoriamento Remoto) - Instituto Nacional de Pesquisas Espaciais, São José dos Campos, INPE: 2000. 171p. 
ZHA, Y.; GAO, J.; NI, S. Use of normalized difference built-up index in automatically mapping urban areas from tm imagery. International Journal of Remote Sensing, v. 24, n. 3, p. 583-594, 2003.

ZHANG, Y.; ODEH, I. O.; HAN, C. Bi-temporal characterization of land surface temperature in relation to impervious surface area, ndvi and ndbi, using a subpixel image analysis. International Journal of Applied Earth Observation and Geoinformation, v. 11, n. 4, p. $256-$ 264, 2009. ISSN 0303-2434. 